\title{
Stochastic Geometry Based Handover Modelling in Two-tier Femtocell Networks
}

\author{
Zoltán Jakó $^{\mathrm{a} *}$, Gábor Jeney ${ }^{\mathrm{a}}$, Joydev Ghosh ${ }^{\mathrm{b}}$, Sanjay Dhar Roy ${ }^{\mathrm{c}}$ \\ ${ }^{a}$ Department of Networked and Services Budapest University of Technology and Economics 1117, \\ Budapest, Magyar tudósok krt. 2., Hungary \\ ${ }^{b}$ Department of Electronics and Tele-communication Engg., The New Horizons Institute of Technology, \\ Durgapur-08, W.B, India
}

${ }^{c}$ Department of Electronics and Communication Engg., National Institute of Technology, Durgapur-09, W.B, India

\begin{abstract}
This paper presents a two-tier cellular structure with femtocells in open access mode. The addition of these base stations extends the offered system capacity and coverage area of the network. This two network parameters nowadays becomes rather important to provide Quality of Service (QoS) for data-hungry applications. Femto base stations (FBS) are deployed randomly to extend the coverage of conventional base stations. Since the femtocells are operating here in open access mode, so that the handover probability parameter becomes important concern. In this article, we declare the handover probability using the fundamentals of stochastic geometry.
\end{abstract}

Keywords: Macrocell; Femtocell; Handover Modelling; Stochastic Geometry; Coverage Probability; Handover Probability.

\section{INTRODUCTION}

The amounts of indoor multimedia services are growing year by year [1]. The $4 \mathrm{G}$ mobile operators are to install additional smaller cells to their traditional cellular structure for the sake of larger capacity, fewer call drops and for better network coverage [2], [3]. The result is a heterogeneous cellular network which contains smaller cells like microcells, picocells or femtocells other than the macrocells. The obvious way to serve indoor coverage demand is to deploy femtocells in highly populated areas. A femtocell usually provides coverage to a smaller area (e. g. flat or house) and transports the data to the femtocell gateway via wired technologies. However, some companies also foresee femtocells as small cells covering outside regions, installed on e. g. lamp, or utilities poles. The biggest advantage of a femtocell over other small cells such as picocell and microcell is that it can be installed by the users. Femtocells can operate in close, open or hybrid mode [4]. In open access mode the connection to the femtocell is allowed for all potential users, meanwhile in close access mode the connection to a femtocell is allowed for a designated group. Hence, an open access femtocells increase the coverage, meanwhile closed mode enhances the quality for those groups. The goal of this paper is to give an analytical model based on stochastic geometry tools and provide an insight on handover probability. The probability of handover at a given location is a basic parameter of several QoS parameter such as call drop ratio or delay. Femtocells do not implement soft handover, thus only hard handover acceptable in this

*Address correspondence to this author at Department of Networked and Services Budapest University of Technology and Economics 1117 , Budapest, Magyar tudósok krt. 2., Hungary; E-mail: \{jakoz, jeneyg\}@hit.bme.hu environment. The hard handover generates a short interruption in the communication, while the handover process to the new base station is completed. This interruption time mostly depends on Round Trip Time (RTT) of the message exchange between the FBS and the femtocell gateway, which is generally, varies on a scale between $10-$ $100 \mathrm{~ms}$ according to [5]. However in $4 \mathrm{G}$ the maximum interruption time is $25 \mathrm{~ms}$ [6]. The handover between two base stations has to be seamless for a user either for a voice calls or latency intolerant multimedia service. Hence, the investigation of handover probability is important. Many paper investigate the coverage probability as QoS parameter in multi-tier cellular networks [7], [8], [9], however, the effect of handover not considered. We found that handover probability for a randomly located user is discussed in [10], however it is based on one tier cellular networks. The goal of this paper is to calculate the handover probability at a randomly located user in a multi- tier environment. We initially defined the Signal-to-Interference ratio (SIR) [11] afterwards, we defined the handover probability. Since the femtocells are operating in open access mode this parameter becomes important due to the strict QoS levels for LTE.

This article is organized as follows: Section 2 shows the system model which is based on the works of [9]. Section 3 describes the SIR and the network coverage, while Section 4 deals with the handover probability. Section 5 illustrates the result, and in the end Section VI concludes the work. 


\section{SYSTEM MODEL}

The system model discussed in this paper is illustrated in Figure 1. MBS is located at the center of a circle cell in the Euclidean plane. Femtocells are randomly deployed according to a Poisson Point Process $\theta$ (PPP), with intensity $\lambda_{f}$. The average numbers of femtocells at the model area are denoted by $N_{f}=\lambda_{f} A$. where A is the area of the model.

Mobile users are distributed uniformly in the area of the model, with a density $\lambda_{u}$. One realization given in Figure 1 . It is assumed that the intensities accept the following inequality: $\lambda_{f}<\lambda_{u}$. We assume that every mobile user connects to the base station that has a strongest received power at the given

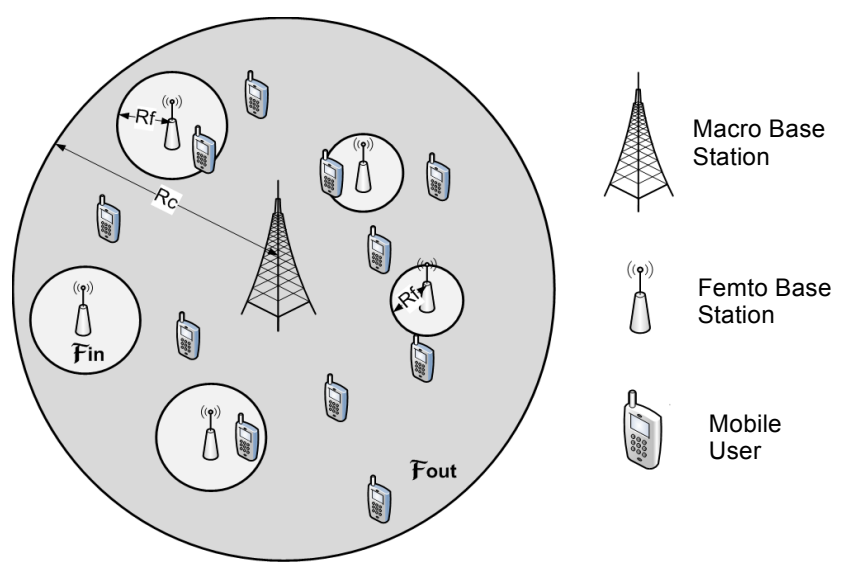

Figure 1. System model

position. Every femtocell operates in open access mode. The coverage area of the macro base station (MBS) is represented by a circle cell. Our system model is assumed to be interference limited, hence the effect of additive noise is neglected. The wall penetration loss is also neglected for the sake of simpli-city. Femtocells and macrocells operate in the same spectra (and frequency reuse equals to 1). The emitted power from the MBSs and femto base stations (FBSs) are denoted by $P_{c}$ and $P_{f}$, respectively. A MBS emits higher power, than a FBS, thus the following assumption is made: $P_{f} \ll P_{c}$. It is assumed that every femtocell has omnidirectional antenna, and each base station covers a $R_{f}^{2} \pi$ circle area as shown in Figure 1, see the light grey circles. Following the idea of [9] the border of the femtocell coverage radius $\left(R_{f}\right)$ is not a constant value. A femtocell coverage area may depend on the distance between the FBS and MBS, the fading, and the transmission power of the base stations. In this paper the femtocell coverage border points are the points where the long-term average received power received from the MBS and the femtocell are the same. In other words, if a given position $(x, y)$ is assumed as a cell boundary on a plain than the received power from the MBS to FBS is equal to

$$
P_{C_{r}}^{(x, y)}=P_{f_{r}}^{(x, y)}
$$

if the coverage radius is shown in the following form (for detailed proof see [9]):

$$
R_{f}=\frac{\left(\frac{P_{c}}{P_{f}}\right)^{1 / \alpha} D}{\left|\left(\frac{P_{c}}{P_{f}}\right)^{2 / \alpha}-1\right|}
$$

where $D$ is the distance between the tagged FBS and the $\mathrm{MBS}, \propto$ denotes the outdoor path loss exponent, and it is assumed that $\alpha \geq 2$.

\section{COVERAGE PROBABILITY}

This section deals with the coverage probability of a randomly located user. The coverage probability denotes the. probability of the SIR level is greater than the threshold level $\gamma$. This is actually the complementary cumulative distribution function $(\mathrm{CCDF})$ of the outage probability:

$$
\mathbb{P}\{S I R>\gamma\}=1-\mathbb{P}\{S I R \leq \gamma\}=1-F(\gamma)
$$

Firstly, we defined the received power a

$$
P_{r}=P_{x} h_{x} g_{x} r_{x}^{-\alpha}
$$

where $x$ can be substituted either by $c$ (for macrocell), or by $f_{i}$ (for the i-th femtocell), $r$ is the distance between the base station and the mobile user, $\alpha$ is the path loss exponent, $g$ represents the effect of slow (lognormal) fading and $h$ denotes the fast fading. We assume that

- the fast fading is Rayleigh fading, to get the worst case scenario (in terms of performance loss). The fast fading has very short correlation properties, more precisely, fading values corresponding to subsequent time frames which regarded as completely independent.

- The slow fading is slow enough to have high correlation of fading values corresponding to subsequent time frames, that they can be regarded equal for $N_{H O}$ frames. We assume that $N_{H O}$ (the number of subsequent measurement time slots before executing a handover) is small enough that this assumption is valid.

Signal-to-interference ratio (SIR) at the mobile user who is served by macrocell base station is given as

$$
S I R_{c}=\frac{P_{c} h_{c} g_{c} r_{c}^{-\alpha}}{P_{f_{j}} h_{f_{j}} g_{f_{j}} r_{f_{j}}^{-\alpha}+\sum_{f, i \neq j}^{i \in \theta} P_{f_{i}} h_{f_{i}} g_{f_{i}} r_{f_{i}}^{-\alpha}},
$$

where the $\mathrm{j}$-th femtocell is the closest one, thus it was expanded from the sum. The effect of the neighboring MBSs is ignored. The interference generated by femtocells can be written as a spatial shot noise process [12]. The SIR at the mobile user, who is served by the $\mathrm{j}$-th FBS equals to 


$$
S I R_{f}=\frac{P_{f_{j}} h_{f_{j}} g_{f_{j}} r_{f_{j}}^{-\alpha}}{P_{c} h_{c} g_{c} r_{c}^{-\alpha}+\sum_{i \in \theta_{f}, i \neq j} P_{f_{i}} h_{f_{i}} g_{f_{i}} r_{f_{i}}^{-\alpha}} .
$$

\section{HANDOVER PROBABILITY}

Handover is executed if the SIR level of the serving cell goes under the SIR level of a neighboring cell in $N_{H O}$ consecutive time slot [10]. Parameter $N_{H O}$ depends on the system, we will keep it as variable for the sake of generality. Handovers between a femtocell and the macrocell (or vice versa) are considered in this paper. Our paper defined three zones of coverage areas:

- $\mathcal{F}_{\text {out }}$ : The zone covered by the macrocell BS, where the probability of femtocell coverage is negligible, thus no handovers could be expected.

- $\mathcal{F}_{\text {in }}$ : The zone covered by the femtocell BS, where the probability that the user handovers to the macrocell is negligible.

- $\mathcal{F}_{\text {HO}}$ : Handover (HO) zone covered by both the femtocell and the macrocell $\mathrm{BS}$, where the handover is probable.

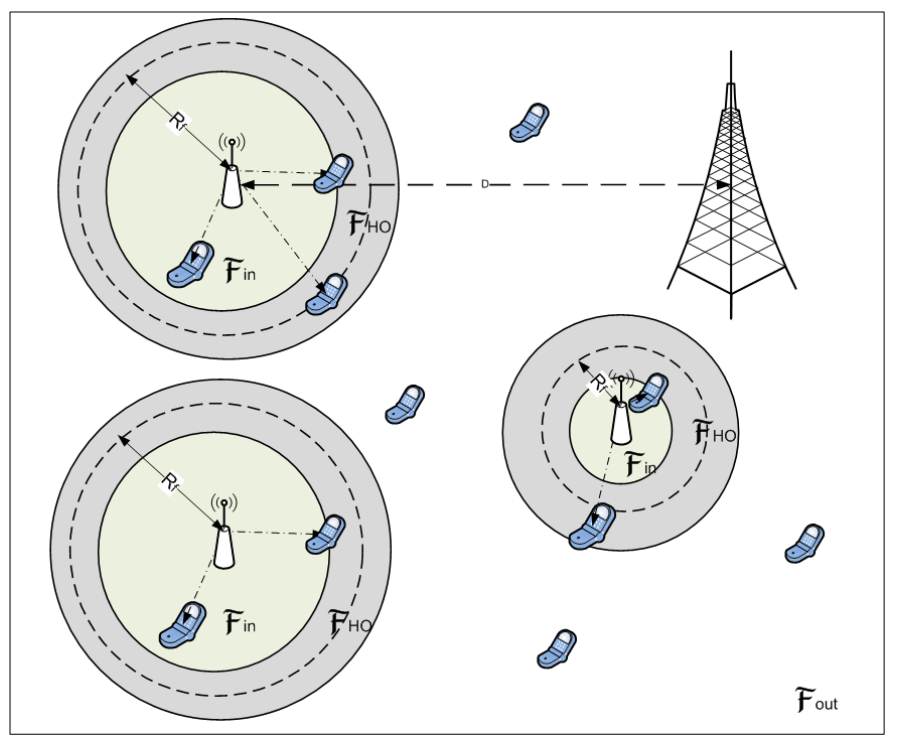

Figure 2. Coverage areas

Usually the F-zone is a concentric ring around the femtocell base stations. Zones are represented by different tones of grey in Figure 2.

The following handover scenarios are considered in this paper:

- A mobile user who is in the HO zone and served by the macrocell handovers to the femtocell,

- A mobile user who is in the HO zone served by the femtocell handovers to the macrocell.
User mobility is not considered in this paper, static user positions are assumed.

The probability of handover can be described as the event that 1) after a successful serving time slot, 2) in $\mathrm{N}$ subsequent time slots, the SIR of the active serving cell drops below the SIR of the other cell. To catch the spirit of the idea, we first take the SIR defined in (2) and (3).

The probability that the SIR of the active serving cell drops below the SIR of the other cell will be denoted by $p$, and can be described as:

$p_{c}=\mathbb{P}\left\{S I R_{c}<S I R_{f}\right\}=\mathbb{P}\left\{P_{c} h_{c} g_{c} r_{c}^{-\alpha}<P_{f_{j}} h_{f_{j}} g_{f_{j}} r_{f^{\prime}}^{-\alpha}\right\}$

where we assumed that the user is served by the $\mathrm{j}$-th femtocell The other case $\left(p_{f}\right)$ could be similarly constructed, only the inequalities change. Thus we will follow only this path. For proof of (4), see Section A in the appendix. Although the slow fading is also random, by nature $g_{c}$ is constant in the short run. The only variable which is changing time slot to time slot, is the fast fading component, i. e. $h_{c}$ and $h_{f}$ :

$$
p_{c}=\mathbb{P}\left\{h_{c}<\left(\frac{P_{f_{j}} g_{f_{j}} r_{f_{j}}^{-\alpha}}{P_{c} g_{c} r_{c}^{-\alpha}}\right) h_{f_{j}}\right\}
$$

where all the (short term) constant terms are collected into one bracket. We well know that Rayleigh distributed amplitudes with parameter $\sigma$ yield exponentially distributed power with 2 parameter $\sigma^{2} / 2$. Assuming Rayleigh parameter $\sigma_{c}$ for the macrocell and $\sigma_{f}$ for the femtocell, we get

$$
p_{c}=\frac{\sigma_{c}^{2} P_{f_{j}} g_{f_{j}} r_{f_{j}}^{-\alpha}}{\sigma_{f}^{2} P_{c} g_{c} r_{c}^{-\alpha}+\sigma_{c}^{2} P_{f_{j}} g_{f_{j}} r_{f_{j}}^{-\alpha}} .
$$

For proof, see Section B in the appendix and [13]. Similarly,

$$
p_{f}=\frac{\sigma_{f}^{2} P_{c} g_{c} r_{c}^{-\alpha}}{\sigma_{f}^{2} P_{c} g_{c} r_{c}^{-\alpha}+\sigma_{c}^{2} P_{f_{j}} g_{f_{j}} r_{f_{j}}^{-\alpha}} .
$$

Please note that if all parameters of the system is known, (6) and (7) could be easily calculated. Note also that $p_{c}+p_{f}=$ 1 always, as expected. Now, the probability of handover could be expressed as a geometrical distribution with parameter $p$ :

$$
\mathbb{P}\{\text { handover }\}=(1-p) p^{N_{\text {Hо }}},
$$

where $p$ could be substituted either by $p_{c}$ or by $p_{f}$. The first term of (8) refers to the time instant before the first SIR 
setback: here the serving base station has sufficiently high SIR. Then, in the next $N_{H O}$ consecutive time slots, (6) must hold, i. e. the SIR should go under the SIR of the other base station-this is given in the last term, assuming independence.

\section{RESULTS AND DISCUSSION}

One possible trajectory of femtocell deployment is given in Figure 3 . The area of the model an $500 \times 500$ square where the MBS is located at the center. The femtocells are spread on the area of the square according to PPP $\theta_{f}$ and the average number of femtocells is $N_{f}=25$. The following parameters are applied for the probability calculation: The macro base station and femtocell emitted power is $P_{c}=$ $20 \mathrm{~W}$ and $P_{f}=20 \mathrm{~mW}$, respectively. The path loss exponent $\alpha=4$. The fast and slow fading components are the same for both macro and femtocell, hence $\sigma_{c}=1$, $\sigma_{f}=1, g_{c}$ and $g_{c}$ follow lognormal distribution with $(0,1)$ parameter. The investigated time slot parameter $N_{H O}$ is equal 3 . The handover probability is given from a macrocell and femtocell user view. The combination of the two handover zone figures are given in Figure 4. Every femtocell has a concentric ring (as we expected), where the handover probability is around $40 \%-60 \%$. The femtocells that located close to the MBS have small coverage zone, meanwhile leaving the MBS the femtocell coverage zone is expanding as defined in (1). It is readable from Figure 4 that the smallest femtocell coverage (in this realization) is has approx. 7 meter radius, meanwhile the largest femtocell coverage radius is approx. 60 meter, which is the same results (1) gives.

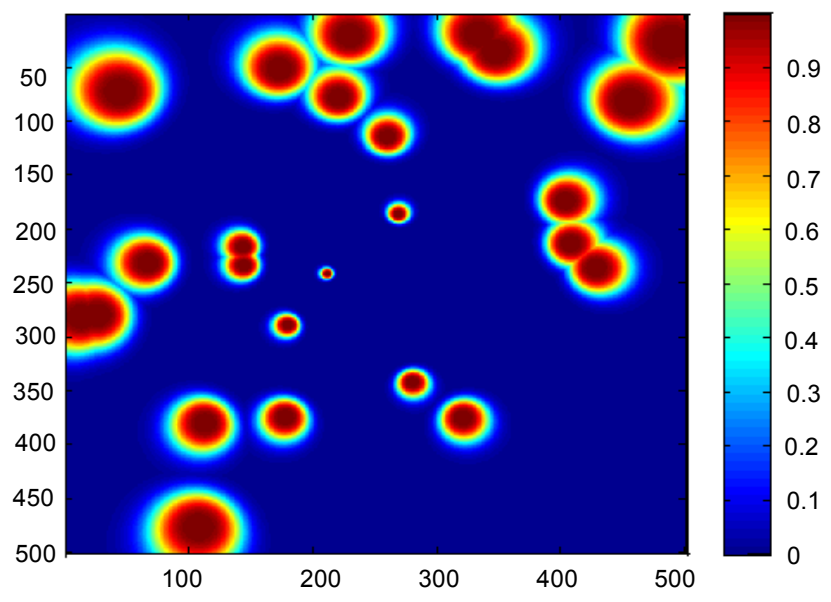

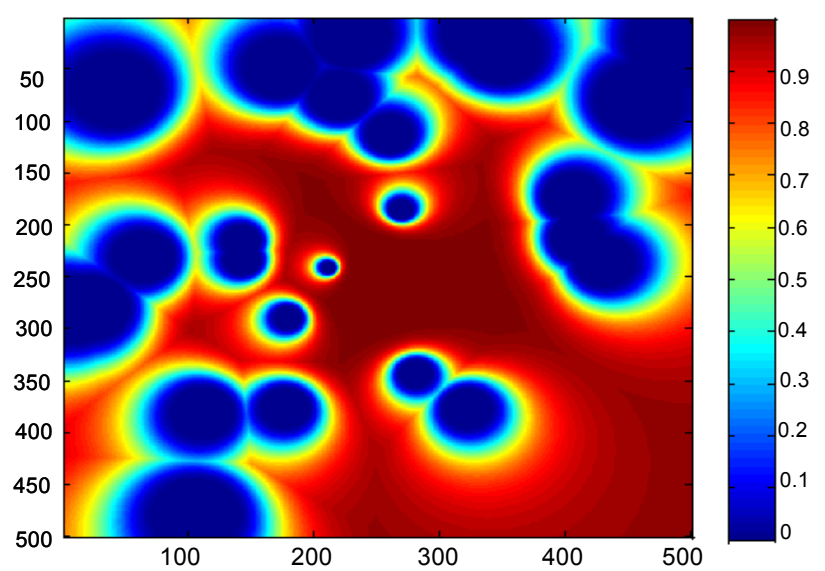

Figure 3. Handover probability from a macrocell or a femtocell user view

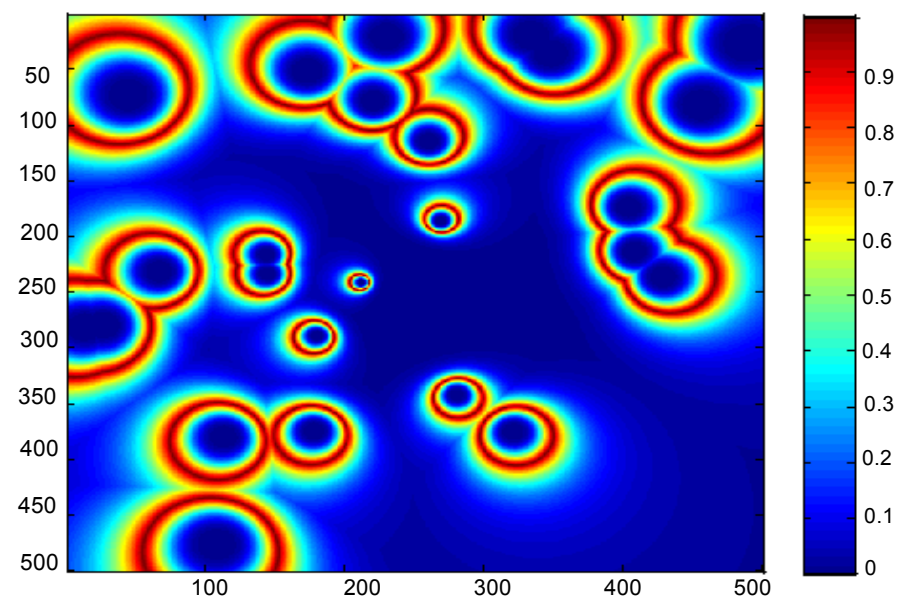

Figure 4. Concentric rings around femtocells where handover is probable

\section{CONCLUSION}

In the paper, an analytical model has been proposed for handover modelling in two-tier femtocell environments based on stochastic geometrical tools. With this model the handover probability is easily calculable. The results shows that the coverage area of the femtocells can be modelled with a circle and the radius of the circle extending while leaving the macro base station. Every femtocell has a concentric ring where the handover is probable. 


\section{APPENDIX}

\section{A. Proof of (4)}

We would like to prove that $\mathbb{P}\left\{S I R_{c}<S I R_{f}\right\}$ yields (4). First, take (2) and rewrite it as $A /(B+C)$ for easier notations. It is clear that (3) equals $B /(A+C)$. Note that all $A, B$ and $\mathrm{C}$ are positive. Thus,

$$
\begin{aligned}
p_{c} & =\mathbb{P}\left\{S I R_{c}<S I R_{f}\right\}=\mathbb{P}\left\{\frac{A}{B+C}<\frac{B}{A+C}\right\} \\
& =\mathbb{P}\left\{\frac{(A-B)(A+B+C)}{(B+C)(A+C)}<0\right\}=\mathbb{P}\{A<B\}
\end{aligned}
$$

which concludes the proof.

\section{B. Proof of (6)}

Starting from (5), one can write

$$
p^{c}=\mathbb{P}\left\{h^{c}<K h^{f}\right\},
$$

where $K$ is constant, $h_{c}$ and $h_{f}$ are both random, following exponential distribution. Using the law of total probability,

$$
p_{c}=\sum_{x} \mathbb{P}\left\{h_{c}<K x \mid h_{f}=x\right\} \mathbb{P}\left\{h_{f}=x\right\} .
$$

Substituting the cumulative probability function and the probability density function of the exponential distribution, the expression yields

$$
p_{c}=\int_{0}^{\infty}\left(1-e^{-\frac{\sigma_{c}^{2}}{2} K x}\right) \frac{\sigma_{f}^{2}}{2} e^{-\frac{\sigma_{f}^{2}}{2} x} \mathrm{~d} x
$$

Further,

$p_{c}=1-\int_{0}^{\infty} \frac{\sigma_{f}^{2}}{2} \exp \left(-\frac{\sigma^{2}+K \sigma_{c}^{2}}{2} x\right) \mathrm{d} x=1-\frac{\sigma_{f}^{2}}{\sigma^{2}+K \sigma_{c}^{2}}$,

which yields (6).

\section{REFERENCES}

[1] Lajos Hanzó, Harald Haas, Sándor Imre, Dominic O’Brien, Markus Rupp, and Laszló Gyöngyösi. Wireless myths, realities, and futures: from 3G/4G to optical and quantum wireless. Special Centennial Issue, Proceedings of the IEEE, 100, pp. 1853-1888, 2012.

[2] Joydev Ghosh, Sanjay Dhar Roy. Qualitative Analysis for Coverage Probability and Energy Efficiency in Cognitive-Femtocell Networks under Macrocell Infrastructure. Electronics Letters, Vol. 51, No. 17,pp.1378-1380,Aug.2015.
[3]Joydev Ghosh, Sanjay Dhar Roy. Implementing a Flexible Testbed Using Dynamic FFR Scheme in OFDMA Based Cognitive Radio Networks. INDICON-2016, India.

[4] Joydev Ghosh, Sanjay Dhar Roy. The Implications of Cognitive Femtocell Based Spectrum Allocation Over Macrocell Networks. Wireless Personal Communications, DOI:10.1007/s11277-016-3597-x,13th Aug'16.

[5] 3GPP TR 25.912 V9.0.0. 3rd Generation Partnership Project; Technical Specification Group Radio Access Network; Feasibility study for evolved Universal Terrestrial Radio Access (UTRA) and Universal Terrestrial Radio Access Network (UTRAN) (Release 9). 2009 More than Six Authors

[6] Rashid A. Saeed, Bharat S. Chaudhari and Rania A. Mokhtar eds. Femtocell Communications and Technologies: Business Opportunities and Deployment Challenges. Hershey: IGI Global, 2012. Print.

[7] Jeffrey G. Andrews, Francois Baccelli and Radha Krishna Ganti. A Tractable Approach to Coverage and Rate in Cellular Networks. IEEE Transactions on Communications, 2011, pp.3122-3134..

[8] Harpreet S. Dhillon, Radha Krishna Ganti, Francois Baccelli and Jeffrey G. Andrews,. Modeling and Analysis of K-Tier Downlink Heterogeneous Cellular Networks. submitted to IEEE JSAC, March. 2011.

[9] H.-S. Jo, P. Xia and J. G. Andrews. Downlink Femtocells Networks: Open or Closed?. IEEE International Conference on Communications, 2011

[10] L. Decreusefond, P. Martins and T. T. Vu. An analytical model for eval- uating outage and handover probability of cellular wireless networks. Submitted, 2011.

[11] Mehdi Monemi, Alireza Zolghadrasli, Shapoor Golbaharhaghighi. High-performance SINR assignment in multi-cell wireless networks. AEU - International Journal of Electronics and Communications Volume 69, Issue 10, October 2015, Pages 1403-1411.

[12] Francois Baccelli and Bartłomiej Błaszczyszyn. Stochastic Geometry and Wireless Networks Volume I., Now Publishers Inc. ISBN-13: 978$1601982643,2009$.

[13] Sunil Kandukuri and Stephen Boyd. Optimal Power Control in Interference-Limited Fading Wireless Channels With Outage-Probability Specifications. IEEE Transactions on Wireless Communications, Vol. 1., No. 1, 2002. 